\title{
Paediatric liver transplantation for children treated at public health facilities in South Africa: Time for change
}

S G Lala, ${ }^{1}$ MB BCh, DCH (SA), DTM\&H, FCPaed (SA), MMed, PhD; R Britz, ${ }^{2}$ MB BCh, DA (SA), FCS (SA); J Botha, ${ }^{2}$ MB BCh, FCS (SA); J Loveland, ${ }^{2}$ MB BCh, FCS (SA), Cert Paed Surg (SA)

${ }^{1}$ Department of Paediatrics, Chris Hani Baragwanath Academic Hospital, University of the Witwatersrand, Johannesburg, South Africa

${ }^{2}$ Transplant Division, Wits Donald Gordon Medical Centre, University of the Witwatersrand, Johannesburg, South Africa

Corresponding author: S Lala (sanjay.lala@wits.ac.za)

Paediatric liver transplantation (PLT) is the only therapeutic option for many children with end-stage chronic liver disease or irreversible fulminant hepatic failure, and is routinely considered as a therapy by paediatric gastroenterologists and surgeons working in developed countries. In South Africa (SA), a PLT programme has been available at Red Cross War Memorial Children's Hospital in Cape Town since November 1991, and another has rapidly developed at the Wits Donald Gordon Medical Centre in Johannesburg over the past decade. However, for most children with progressive chronic liver disease who are reliant on the services provided at state facilities in SA, PLT is not an option because of a lack of resources in a mismanaged public health system. This article briefly outlines the services offered at Chris Hani Baragwanath Academic Hospital - which is typical of state facilities in SA - and proposes that resources be allocated to establish an innovative, nationally funded centre that would enable greater numbers of children access to a PLT programme.

S Afr Med J 2014;104(11):829-832. DOI:10.7196/SAMJ.8624

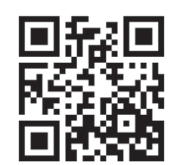

The Department of Paediatrics at Chris Hani Baragwanath Academic Hospital (CHBAH), Johannesburg, South Africa (SA), offers, among others, a combined gastroenterology, hepatology and nutrition service. A weekly outpatient clinic is staffed by three paediatric gastroenterologists, all of whom have major clinical responsibilities in multiple general paediatric wards. This clinic sees $\sim 650$ patients per year.

Most of our patients' families come from impoverished backgrounds and are reliant on our services to provide medical care for their children. Although our unit predominantly serves children residing in Gauteng province, we care for children residing in North West and Limpopo provinces and KwaZulu-Natal. Within Johannesburg, the unit cares for children with liver disease who were originally treated at various district and regional hospitals, as well as the academic complexes based at Charlotte Maxeke Johannesburg Academic and Rahima Moosa Mother and Child hospitals. Each year, we see scores of children with chronic liver disease, predominantly secondary to biliary atresia. Sadly, only a handful of children receive liver transplantation, and selecting which children are to receive a liver transplant is perhaps the greatest ethical and moral dilemma that confronts paediatricians working in the government sector. 


\section{What are the major difficulties in treating children with chronic liver disease at government hospitals?}

With the likely exception of state facilities in the Western Cape province, the steady deterioration of services at government hospitals is well documented, ${ }^{[1]}$ and $\mathrm{CHBAH}$, despite being a major academic teaching hospital in SA, is no different. There are neither full-time paediatric gastroenterologists nor full-time paediatric gastroenterology nurses, who are needed to cope with the medical needs of the hundreds of children who attend our clinic. Our outpatients and inpatients have to share overcrowded and understaffed facilities; in particular, we have no isolation facilities to prevent exposure to highly infectious pathogens such as respiratory viruses and tuberculosis (TB). There are also perennial problems with the regular provision of essential medications, and we do not have a reliable laboratory service. The hospital cannot afford to provide nutritional supplementation to the majority of our paediatric patients, and most parents cannot afford to purchase additional nutritional supplements.

\section{How do we approach transplantation?}

In the past, so-called state patients were referred to Red Cross War Memorial Children's Hospital (RCWMCH) in Cape Town, which was the only centre performing paediatric liver transplantation (PLT). However, RCWMCH has very limited capacity and cannot offer liver transplantation to the vast majority of children managed at government facilities; only a handful of PLTs are performed per year. Over the past decade, the development of a world-class transplant facility and the availability of living related donor (LRD) transplantation at Wits Donald Gordon Medical Centre (WDGMC a private academic hospital associated with the University of the Witwatersrand) have significantly improved the numbers of children receiving liver transplants. This development has, however, placed pressure on paediatric gastroenterologists working in government hospitals to refer children for liver transplantation. This increasing pressure is related in part to the time and resources needed to evaluate and refer potential recipients for transplantation. Nonetheless, despite the availability of this surgical expertise, there is no capacity to provide adequate lifelong medical care at $\mathrm{CHBAH}$ following transplantation. Currently, the paediatric nephrology service at Charlotte Maxeke Johannesburg Academic Hospital has been providing post-transplant follow-up care for the paediatric liver transplant recipients who have been treated at state facilities.

In SA, there is no reliable national registry describing the aetiology of liver disease in children who are potential candidates for liver transplantation. At CHBAH, a failed hepatoportoenterostomy procedure (Kasai procedure) in children with biliary atresia accounts for the vast majority of referrals for paediatric liver transplantation. Children with metabolic liver disease (such as a1-antitrypsin deficiency, tyrosinaemia and Wilson disease) and other cholestatic disorders (such as Alagille syndrome and progressive intrahepatic cholestatic syndrome) are likely to account for the remainder of the referrals for liver transplantation. Although acute liver failure accounts for about a tenth of paediatric liver transplantation performed in developed countries, ${ }^{[2]}$ our experience at $\mathrm{CHBAH}$ suggests that the dire lack of paediatric intensive care facilities will severely restrict the number of children with acute liver failure who can be referred for transplantation.

How then do we approach the issue of liver transplantation for children who are treated in government hospitals? Although we consider each child on an individual basis, we currently use the following general approach:
- If there are no medical or psychosocial contraindications to transplantation, we refer the child to WDGMC and/or RCWMCH. If the family has medical insurance, we tend to refer the child to WDGMC because of the greater likelihood of receiving a transplant (either from a deceased donor or LRD).

- If there are medical or psychosocial contraindications to transplantation, we manage the medical complications attributable to chronic liver disease as best we can, and we aim to provide as much holistic care as possible, taking into account the limited resources available in the state sector. During this time, we continually reassess the medical or psychosocial circumstances, and if these improve or stabilise, we reconsider referring the child for transplantation.

Previously, most of our paediatric patients have not been referred for transplantation because of adverse social or financial circumstances, and the inadequate resources available in government hospitals to deal with these problems. More recently, several of our paediatric patients (including patients without medical insurance) have been referred to, listed for and successfully transplanted at WDGMC using either deceased donor or LRD grafts. If more resources were made available at government hospitals for paediatric patients requiring liver transplantation, many more patients would be receiving this lifesaving procedure. For example, we estimate that five additional children would be referred for liver transplantation from $\mathrm{CHBAH}$ annually should additional resources become available. In Gauteng, it is very likely that a similar number of children who are treated at Steve Biko Academic Hospital would also be referred for transplantation. It is important to note that many clinicians working at other government hospitals do not consider transplantation in children with end-stage chronic liver disease because of real and perceived difficulties in referring children for these lifesaving procedures. Additionally, as increasingly successful hepatoportoenterostomy procedures improve survival rates in children with biliary atresia, the number of children who would require liver transplantation would increase.

\section{The way forward}

The allocation of limited resources to fund a PLT programme in SA, a country with a heavy HIV and TB burden and growing epidemics of non-communicable diseases, high injury and violence levels, and maternal and neonatal deaths, ${ }^{[3]}$ will inevitably pose complex and difficult ethical problems. ${ }^{[4]}$ However, noting the major improvements in health service delivery that have been overseen by the current Minister of Health, Dr Aaron Motsoaledi, and that SA spends $\sim 4.1 \%$ and $4.5 \%$ of its gross domestic product on public and private health provision, respectively, ${ }^{[5]}$ we believe that resources can be made available to fund a national PLT programme. The present SA government has repeatedly committed itself to providing national health insurance to guarantee universal health coverage for all SA citizens. The provision of a PLT programme can be achieved within this framework, provided that there is sufficient political support to drive this process forward, especially with regard to improving leadership and management skills at local state facilities so that resources are effectively utilised.

An effective method for funding a PLT programme could be through a nationally funded centre (NFC) programme, such as exists in Australia. ${ }^{[6]}$ The Australian NFC programme is 'designed to ensure that there is maximal access to certain high-cost, low-demand, new and emerging technologies ... in the context of workforce and resource availability; these technologies are provided efficiently and effectively; and that health and cost outcomes of these technologies are monitored and evaluated. ${ }^{\text {'[6] }}$ 
All potential recipients of PLT should be

entered into a database where standard clinical,

demographic, socioeconomic, and health status

of caregivers is collected. Initiate discussions

about PLT with caregivers. A standardised

information sheet should be given to all caregivers

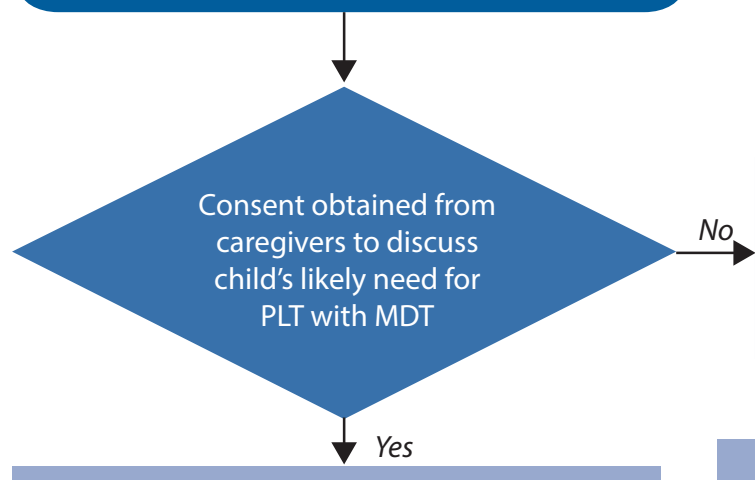

Discuss child's condition and prognosis with MDT

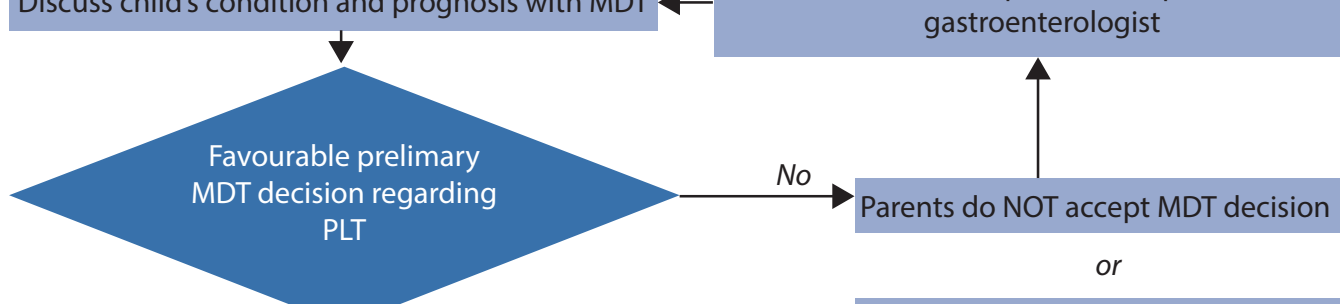

Accept caregivers' decision, continue

supportive medical care and initiate

palliative care if needed

Reconsider referral for PLT if caregivers

change decision, but inform caregivers of

risks of delaying PLT

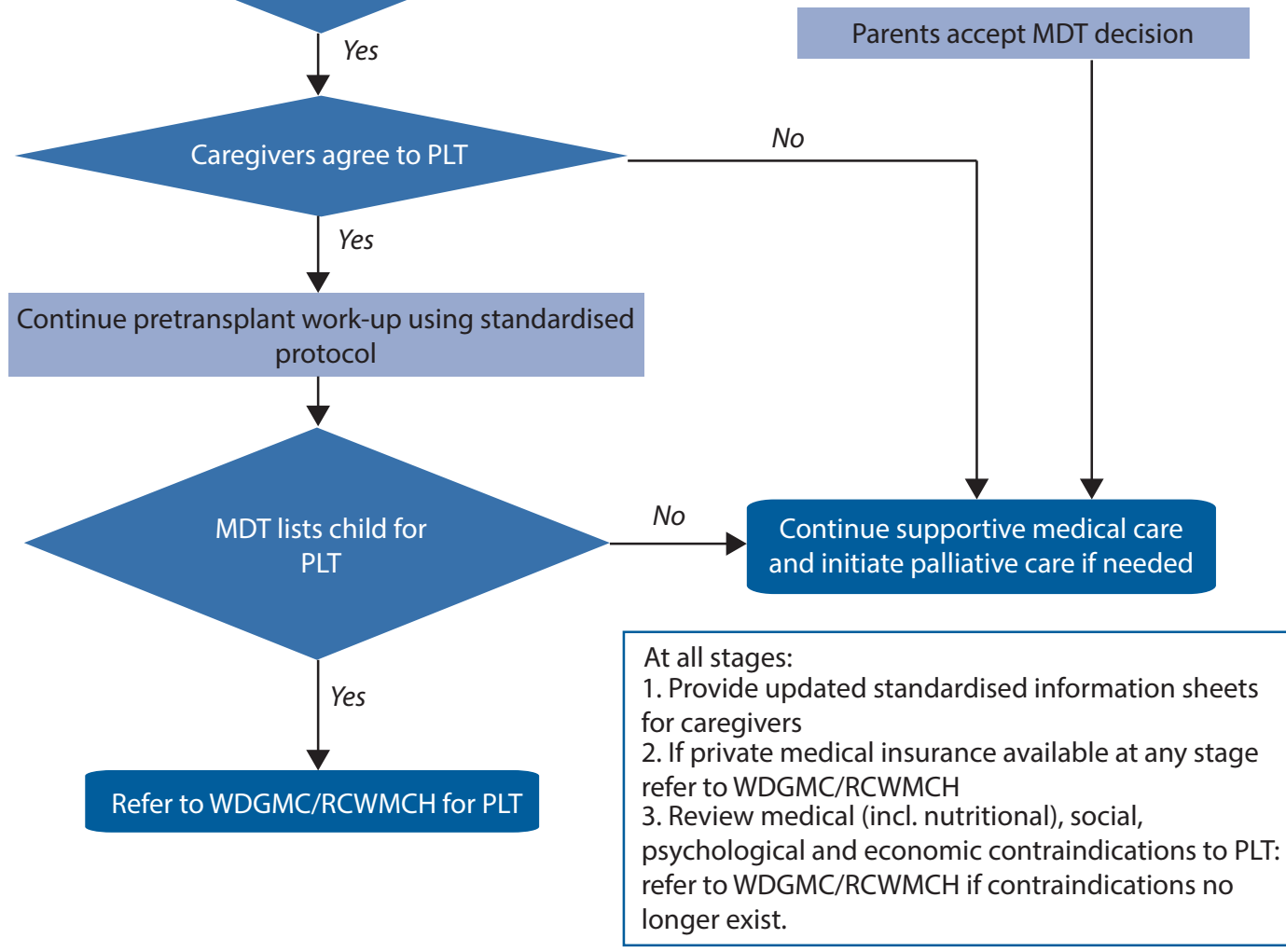

Fig. 1. Suggested referral pathway for paediatric liver transplantation for children treated at state facilities in State. (PLT = paediatric liver transplant; MDT = multidisciplinary team; WDGMC = Wits Donald Gordon Medical Centre; $R C W M C H=$ Red Cross War Memorial Children's Hospital.)

In SA, an NFC programme for PLT is feasible because end-stage chronic liver disease, mostly caused by biliary atresia, is a relatively rare condition in children. There have been previous appeals for a nationally funded liver transplantation programme, ${ }^{[7]}$ and the use of standard global costs to fund liver transplantation and follow-up visits (including the cost of immunosuppressive therapy) at WDGMC should provide further impetus to the national health authorities to implement an NFC programme in SA. ${ }^{[8]}$ 


\section{How do we run a PLT programme for children treated in state facilities in SA?}

Fig. 1 outlines our suggested PLT programme. Although a preliminary report suggests that the incidence of biliary atresia in Soweto may be among the highest in the world, ${ }^{[9]}$ there is a paucity of epidemiological data on children with end-stage chronic liver disease and rapidly progressive acute liver failure in SA (with the exception of a cohort of children who have undergone liver transplantation in Johannesburg and Cape Town). ${ }^{[10,11]}$ We therefore urgently propose that a national register or database be established to determine the number of children, irrespective of their social background or nationality, who potentially require PLT in SA. This register should also reflect the total number of children listed for PLT throughout SA, as well as the number of deaths in children waiting for a PLT.

In addition to clinical data (including the health status of caregivers), information regarding housing, sanitation, electricity, piped water and access to basic education and social grants should be recorded. The attending paediatrician or paediatric gastroenterologist should initiate discussions about the possibility of PLT at the earliest opportunity and gauge the opinions of the caregivers and extended family regarding PLT; this is especially important in SA, as many caregivers often make decisions only after family elders and other extended family members have been consulted. Information sheets, translated into the relevant indigenous languages, should be made available to all families. After obtaining consent from the caregivers, all potential PLT candidates should be considered by a multidisciplinary team (MDT). An MDT would usually comprise surgeons, gastroenterologists/hepatologists, intensive care specialists, liver transplant co-ordinator(s), social workers, dieticians, physiotherapists, psychologists, and nurses this is the current practice followed at the RCWMCH and WDGMC transplant units.

If a PLT is deemed possible by both the caregivers and the MDT, then a pretransplant work-up should be initiated by the referring doctor. Even though there are limited resources in state facilities, most of the pretransplant work-up can take place in state facilities, and investigations not available at the state hospital can be performed at the transplant unit. The clinical and cost effectiveness of protocols used for pretransplant work-ups should be constantly monitored and evaluated. Ideally, the costs of the pretransplant work-up should be borne by the referring hospital, even if parts of the work-up are performed at a government academic hospital or national centre. When considering funding allocation for an NFC programme, it is important to note that inter-provincial monetary payments are not routinely made to the liver transplantation units at RCWMCH and WDGMC for children requiring transplant work-up and who reside outside of the Gauteng and Western Cape provinces.

Facilities to perform PLT and offer perioperative care are well established at WDGMC and RCWMCH and these should not be duplicated because they are already sufficient. Additional paediatric gastroenterologists and hepatologists have recently been trained, but the issue of offering lifelong post-transplant care at state facilities is perhaps the most immediate problem that needs to be addressed. Post-transplant care includes the regular provision of chronic medication, reliable and dependable laboratory and radiological facilities, and the availability of isolation facilities should children who have undergone liver transplantation be readmitted to hospital. We propose that this care, which should be available at regional and academic state facilities, be funded by an NFC programme. If these facilities are not available at state facilities, funding should be provided to cover the costs of admission and investigations performed at WDGMC or RCWMCH. In the long term, at least two stand-alone units with a complement of full-time staff should be established at state academic hospitals to provide lifelong posttransplant care to children reliant on state facilities. The NFC should fund at least two units: a newly established unit in Gauteng and the existing unit at RCWMCH. The existing facilities at RCWMCH should be further strengthened to care for children residing in the Western, Eastern and Northern Cape and Free State provinces. In Gauteng, Nelson Mandela Children's Hospital, currently under construction in Johannesburg, would be an ideal location for a unit serving the children residing in the populous regions of Gauteng, KwaZulu-Natal, Limpopo, Mpumalanga, North West and Free State provinces. Annually, these NFC units should be able to care for $12-14$ and $7-8$ children undergoing transplantation at the WDGMC and RCWMCH, respectively.

Importantly, an NFC programme for PLT needs regular monitoring and evaluation to ascertain the safety and quality of liver transplantation, to review costs associated with PLT and to provide future cost estimates. Teaching and training are essential components of an NFC programme, and research reporting both clinical and quality-of-life outcomes in children undergoing liver transplantation is critical. ${ }^{[6]}$

\section{Conclusion}

The care of children requiring PLT who are treated at state facilities can no longer be ignored when world-class facilities that offer lifesaving surgical procedures are readily available. We appeal to the Minister of Health and relevant health authorities to urgently consider the establishment of an NFC programme that would provide children treated at state facilities access to lifesaving PLT.

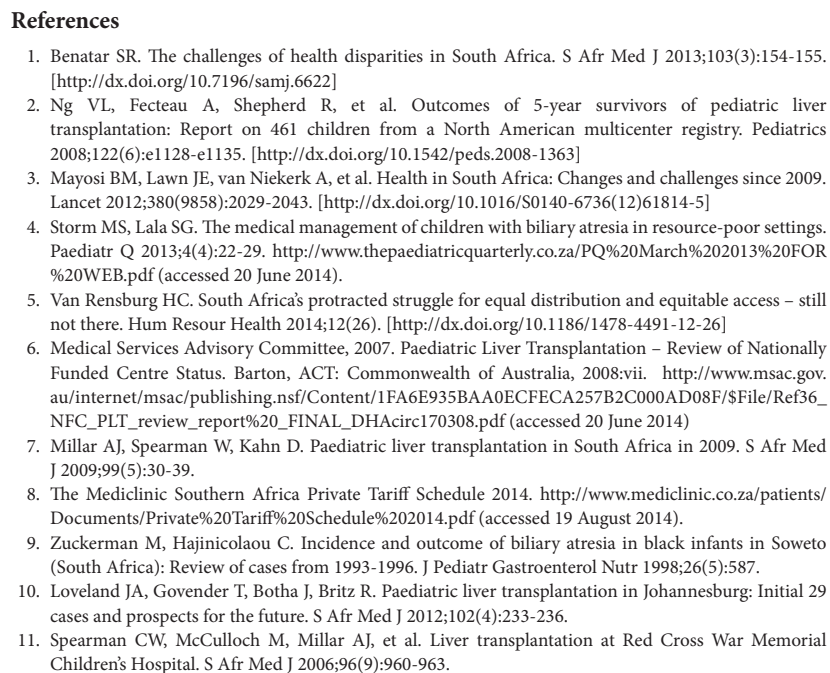

1. Benatar SR. The challenges of health disparities in South Africa. S Afr Med J 2013;103(3):154-155 [http://dx.doi.org/10.7196/sami.6622

2. Ng VL, Fecteau A, Shepherd R, et al. Outcomes of 5-year survivors of pediatric liver transplantation: Report on 461 children from a North American multicenter registry. Pediatrics 2008;122(6):el128-e1135. [http://dx.doi.org/10.1542/peds.2008-1363]

Mayosi BM, Lawn JE, van Niekerk A, et al. Health in South Africa: Changes and challenges since 2009. Lancet 2012;380(9858):2029-2043. [http://dx.doi.org/10.1016/S0140-6736(12)61814-5]

4. Storm MS, Lala SG. The medical management of children with biliary atresia in resource-poor settings. Paediatr Q 2013;4(4):22-29. http://www.thepaediatricquarterly.co.za/PQ\%20March\%202013\%20FOR \%20WEB.pdf (accessed 20 June 2014).

5. Van Rensburg HC. South Africa’s protracted struggle for equal distribution and equitable access - still not there. Hum Resour Health 2014;12(26). [http://dx.doi.org/10.1186/1478-4491-12-26]

6. Medical Services Advisory Committee, 2007. Paediatric Liver Transplantation - Review of Nationally Funded Centre Status. Barton, ACT: Commonwealth of Australia, 2008:vii. http://www.msac.gov. au/internet/msac/publishing.nsf/Content/1FA6E935BAA0ECFECA257B2C000AD08F/\$File/Ref36 NFC_PLT_review_report\%20_FINAL_DHAcirc170308.pdf (accessed 20 June 2014)

7. Millar AJ, Spearman W, Kahn D. Paediatric liver transplantation in South Africa in 2009. S Afr Med J 2009;99(5):30-39.

The Mediclinic Southern Africa Private Tariff Schedule 2014. http://www.mediclinic.co.za/patients Documents/Private\%20Tariff\%20Schedule\%202014.pdf (accessed 19 August 2014).

9. Zuckerman M, Hajinicolaou C. Incidence and outcome of biliary atresia in black infants in Soweto (South Africa): Review of cases from 1993-1996. J Pediatr Gastroenterol Nutr 1998;26(5):587.

10. Loveland JA, Govender T, Botha J, Britz R. Paediatric liver transplantation in Johannesburg: Initial 29 cases and prospects for the future. S Afr Med J 2012;102(4):233-236

Spearman CW, McCulloch M, Millar AJ, et al. Liver transplantation at Red Cross War Memorial Children's Hospital. S Afr Med J 2006:96(9):960-963. 